\title{
Counting on proteomics
}

\section{Download a trial of image analysis software, and seek those proteins.}

\section{ProteomeWorks}

Micromass

www.micromass.co.uk

Proteomics products from an alliance

between BioRad and Micromass

BioRad's PDQuest image analysis software achieves rapid imaging of $2 \mathrm{D}$ gels, and the identification of protein spots of interest. The automated excision and enzymatic digestion of these protein spots for mass spectrometry (MS) analysis have been rate-limiting steps. The ProteomeWorks Spot Cutter is designed to eliminate the first of these two bottlenecks . Driven directly from PDQuest software, it enables automated spot excision and deposition into a microtitre plate (MTP). The excised protein spots can be robotically de-stained, chemically modified and digested in preparation for either MALDI-TOF or ESI mass spectrometry with Micromass MassPREP Station. The MassPREP Station is the robotic proteinhandling system developed for The ProteomeWorks System. Incorporating Windows $\mathrm{T}$ based applications, the MassPREP Station includes a range of standard sample preparation and digest protocols, and user-customization of the standard protocols or the scripting of novel protocols is also supported.

Reader Service No. 100

\section{Ettan MALDI-ToF}

Amersham Pharmacia www.apbiotech.com Adding MS to proteomics systems

The Ettan MALDI-ToF mass spectrometer for protein identification was introduced earlier this year at Pittcon. The system's software matches proteins against known databases using a combination of Prowl from ProteoMetrics LLC and control software developed with Scientific Analysis Instruments Ltd. Another feature of the MALDI-ToF system is the quadratic field reflection technology (Z2) designed with Peter Derrick of Warwick University and Scientific Analysis Instruments. Amersham Pharmacia are planning a series of proteomics launches through the year, including an integrated data management system developed with Cimarron Software to track samples through the proteomics process from 2D electrophoresis to MS.

Reader Service No. 101

\section{MiniSpin}

Eppendorf www.eppendorf.com

\section{'Futuristic'-looking minicentrifuges}

MiniSpin and MiniSpin plus microcentrifuges are suitable for general lab use. When loaded with $1.5 \mathrm{ml}$ or $2.0 \mathrm{ml}$ Eppendorf tubes, MiniSpin is capable of a maximum speed of 13,400 r.p.m., equivalent to $12,100 g$. Equipped with a black aluminium rotor, MiniSpin plus can reach 14,500 r.p.m. $(14,000 g)$. Prominent features of MiniSpin include rapid acceleration, a maximum braking time of 13 seconds, low noise levels and a footprint that is smaller than a sheet of A4 paper. MiniSpin plus has a choice between the short-spin function or continuous operation, as required, for example, for operation in a cold room.

Reader Service No. 102

SwellGel

Pierce www.piercenet.com

Protein purification made fast

SwellGel Nickel Chelating Discs bridge the gap between coated microwell plates and larger column purification. When used for performing high-throughput fusion protein purification using the $6 \times$ His tag, the SwellGel Discs bind more than $1 \mathrm{mg}$ per well, equivalent to spin column capacity in a high-throughput format. The discs are easy to use - precharged, pre-equilibrated and pre-dispensed. Just add clarified lysate to dried media and it is ready to purify without the need to dispense media into 96-well plates. The disc rehydrates to a nickel-chelating agarose gel within $90 \mathrm{sec}$ onds of adding the protein. There's no need to use expensive robotics to ensure even slurry dispensing.

\section{Reader Service No. 103}

\section{ExpressDetector}

KPL

Nickel-labelled HRP to detect His-tagged proteins

KPL's new ExpressDetector System is a simple, direct method for detecting low nanogram levels of recombinant histidine-tagged fusion proteins using nickel-labelled horseradish peroxidase. This highly specific reagent simplifies the process by eliminating the need for primary and secondary antibodies. It offers greater sensitivity on a western blot in less time than monoclonal antihistidine antibodies.

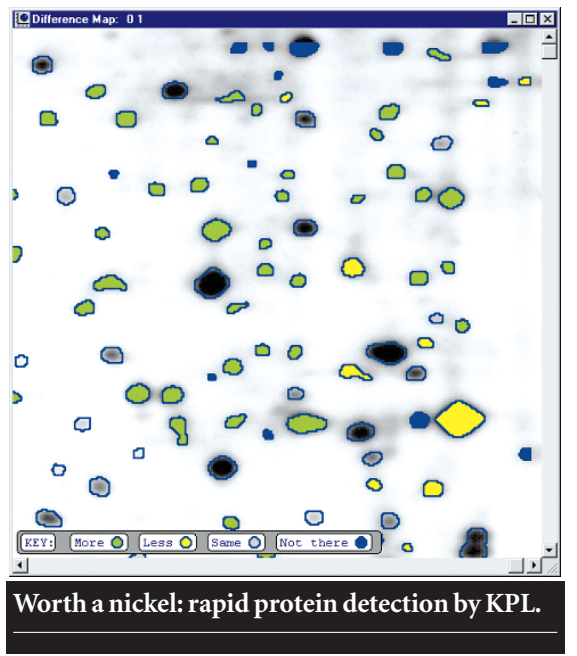

This rapid method is recommended for use in ELISA and blotting. The reagent is offered as conjugate or in the ExpressDetector Chemiluminescent Blotting Kit, with all the components necessary for detection of his-tagged proteins on blots.

Reader Service No. 104

\section{AlphaMatch}

Alpha Innotech www.alphainnotech.com Spot those proteins

AlphaMatch 2D image analysis software is designed for the fully automated identification, analysis and matching of protein spots in a two-dimensional gel image. The software handles molecular weight determination, density calculation, normalization, and various background subtraction methods. The user can identify proteins that share expression changes, search using a wide variety of matching criteria, and edit the results automatically or manually. AlphaMatch 2D allows importation of 8-, 12- or 16-bit tiff images from a variety of imaging sources, and the database option allows for complex queries across a great number of experiments.

Reader Service No. 105

\section{Auto 2-D TDGS}

C.B.S. Scientific

www.cbsscientific.com Automatic two-dimensional gene scanning systems

The Auto 2-D TDGS combines the efficiency of multiplex PCR with the high resolution of two-dimensional denaturing gradient gel electrophoresis. TDGS is intended for screening large numbers of individual DNA samples for mutations and polymorphisms in very large or multiple genes. It is an automated 2-D electrophoresis system that consecutively 
resolves DNA fragment mixtures on the basis of both size (1-D) and base pair sequence (2-D) within the same gel without manual interference. The sample is first separated horizontally and then the electric field reoriented so that separated fragments enter a contiguous vertical U/F gradient to reveal characteristic melting points of each fragment. The multiplex PCR protocol permits co-amplification of as many as 24 fragments in a single reaction, while 2-D electrophoresis can easily resolve about 40 fragments with an average size of $300 \mathrm{bp}$ (including the GCclamp) in 6 hours.

Reader Service No. 106

\section{Undercounter freezer}

Harris www.harrisphq.com

Cold storage, close at hand

Harris, part of the GS Laboratory Equipment group, now offers $-8{ }^{\circ} \mathrm{C}$ low-temperature storage in a space-saving undercounter design. The new 5.0-cubic-foot Essential Undercounter Freezer combines the latest in vacuum insulation panel technology with conventional high-density foamed-in-place urethane insulation. The composition wall maintains insulation $R$-value while decreasing wall thickness from $12.7 \mathrm{~cm}$ to $5 \mathrm{~cm}$. Insulation panels are constructed of INSTILL vacuum insulation core encapsulated by a sealed and evacuated film laminate wrapped in Mylar. The low-profile cabinet is 32.1 inches high, 30.2 inches front-to-back and 41.8 inches wide.

Reader Service No. 107

Adeno-X

Clontech Laboratories www.clontech.com Ligation-based expression system for highlevel protein expression in mammalian cells

The Adeno-X system rapidly produces recombinant adenovirus with greater ease and reliability than existing approaches. The ligationready adenoviral vector contains restriction enzyme sites that render plaque purifications unnecessary, so recombinant adenovirus can be generated within 2-3 weeks. Adenovirus efficiently infects both dividing and nondividing cells, including most human cell types and cells from many other species. This broad range of target cells makes the Adeno-X System suitable for gene function analysis, gene therapy development, antisense therapy, vaccine development, and some transgenic animal studies.

Reader Service No. 108

Imaging systems

Perkin-Elmer www.wallac.com

Imaging for high throughput

EG\&G's advanced imaging systems for highthroughput screening applications, molecular and cell biology, genomics, proteomics

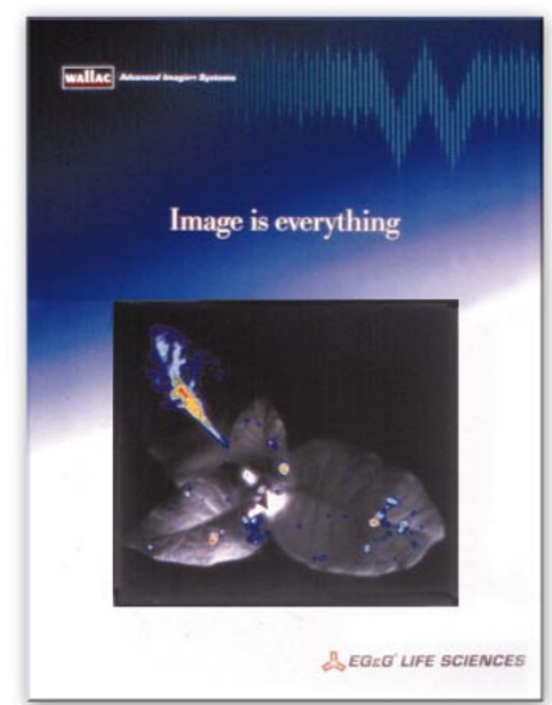

Name change: go to Wallac's website for EG\&G.

and forensics, are now part of the PerkinElmer life sciences range. Included in the product range are the Wallac ARTHUR multi-wavelength fluoroimager and Berthold NightOWL imager for low-light luminescence applications, and a range of application software.

Reader Service No. 108

\section{UltraSNAP}

Vector Laboratories www.vectorlabs.com Detection systems for nucleic-acid blots

The UltraSNAP (Biotin) Detection System utilizes alkaline phosphatase and the DuoLuX substrate for the chemiluminescent or fluorescent detection of biotinylated DNA/RNA probes. The reagents are formulated for high sensitivity with low background in applications such as Southern or northern hybridization, dot blotting, or plaque and colony screening. The UltraSNAP Detection System contains sufficient reagents for the development of approximately $20100-\mathrm{cm}^{2}$ blots.

Reader Service No. 109

\section{AutoGenprep 3000}

a1Biotech

www.a1-biotech.com

Full automation for nucleic-acid

isolation

The latest in the a1-Biotech range of self-contained nucleic-acid isolation systems, AutoGenprep 3000 is a fully automated robotic instrument-chemistry system, developed for the isolation of genome DNA and RNA from various animal origins. al say that this machine produces high-quality nucleic acids with yields comparable to manual methods. The system can process 48 samples per run and 24 samples at a time. Typical processing times are 6.5 hours for 48 samples of whole blood and 3.7 hours for 48 samples of Proteinase-K-pretreated tissue. The AutoGenprep 3000 automatically produces isolates ready for downstream processes, such as BAC end sequencing, fluorescence and capillary sequencing, PCR, RT-PCR, transfections, Southern blotting and northern blotting.

Reader Service No. 110

\section{TotalLab software}

TotalLab

www.totallab.com

Evaluation copy of image analysis software, on the web

TotalLab's new website now contains a free, working copy of the company's software which can be used to analyse customer's own images for evaluation purposes. TotalLab is image analysis software for analysis of $1 \mathrm{D}$ electrophoresis gels and 2D spots, dot and slot blots, other arrays and colonies.

Reader Service No. 111

These notes are compiled in the Nature office from information provided by the manufacturers. For more details, fill in the reader service card
ADVERTISEMENTS

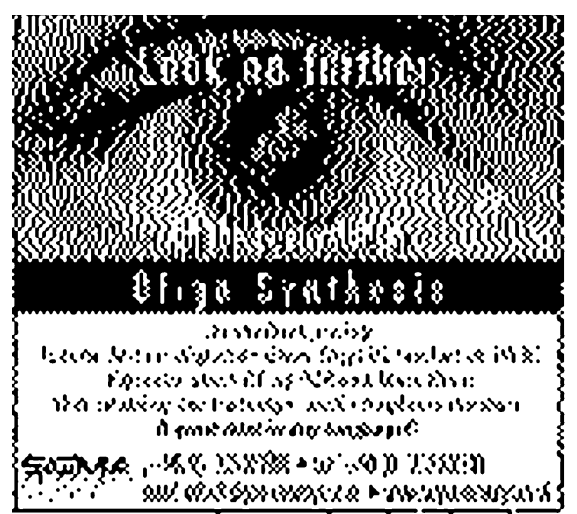

READER ENQUIRY NO. 33

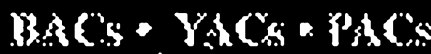

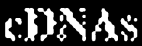

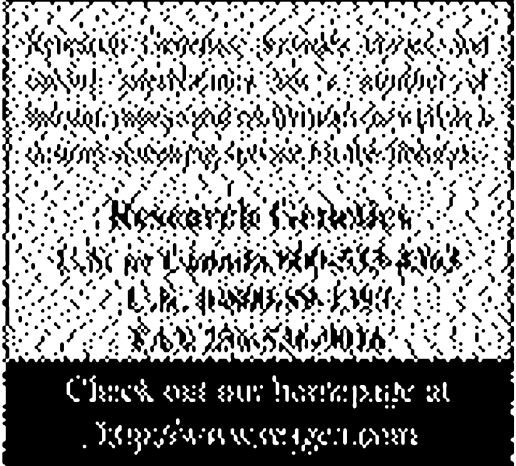

READER ENQUIRY NO. 40 